\title{
A Comparative Study between Bandelet and Wavelet Transform Coupled by EZW and SPIHT Coder for Image Compression
}

\author{
Beladgham Mohammed \\ Department of Electronic, Bechar University, Bechar, Algeria \\ beladgham.tlm@gmail.com \\ Habchi Yassine \\ Depart ment of Electronic, Bechar University, Bechar, Algeria \\ habchi8article@gmail.com \\ Moulay Lakhdar Abdelmouneim \\ Department of Electronic, Bechar University, Bechar, Algeria \\ moulaylakhdar78@yahoo.fr \\ Taleb-Ahmed Abdelmalik \\ Biomecanic Laboratory, Valencienne University, France \\ taleb@univ-valenciennes.fr
}

\begin{abstract}
Second generation bandelet transform is a new method based on capturing the complex geometric content in image; we use this transform to study medical and satellite image compressed using the bandelet transform coupled by SPIHT coder. The goal of this paper is to examine the capacity of this transform proposed to offer an optimal representation for image geometric. We are interested in compressed medical image, In order to develop the compressed algorithm we compared our results with those obtained by the bandelet transform application in satellite image field. We concluded that the results obtained are very satisfactory for medical image domain.
\end{abstract}

Index Terms - Bandelet transform, Compression, Optical flow, Quadtree segmentation, SPIHT and EZW coder .

\section{INTRODUCTION}

Today, distance diagnostic medical system, digital library and Internet are applied popularly. One of the most important problems in such applications is how to store and transmit images [1]. The medical diagnostic field is interested by the researchers. It is well established that the accuracy and precision of diagnostic are initially related to the image quality.

Finding efficient geometric representations of images is a central issue in improving the efficiency of image compression. Many ideas have already been studied to find new bases can capture geometric regularity image. Image representations in separable orthonormal bases such as Fourier, local Cosine or Wavelets can not take advantage of the geometrical regularity of image structures. Standard wavelet bases are optimal to represent functions with piecewise singularities; however, they fail to capture the geometric regularity along the singularities of edges or contours because of their isotropic support. To exploit the anisotropic regularity along edges, the basis must include elongated functions that are nearly parallel to the edges. Multi-scale geometric analysis (MGA) developed recently provides a group of new bas is that has anisotropic supports such as Curvelets [2-7], A Curvelet frame is composed of multiscale elongated and related wavelet type functions, for this reason, In this paper, we introduce a new type of transform, called bandelet transform by E Le Pennec and Stéphane Mallat [8], this transform is more recently developed method of compression technique, which decompose the image along mult iscale vectors that are elongated in the direction of a geometric flow. This geometric flow indicates directions in which the image gray levels have regular variations; bandelet bases can represent the geometric regular images efficiently.

\section{THE BA NDELET TRANSFORM}

Bandelet transform, introduced by Le Pennec and Mallat [9] built a base adapted to the geometric content of an image. The bandelets are obtained from a local deformation of space to align the direction of regularity with a fixed direction (horizontal or vertical) and is reduced to a separable basis [10], [11]. 

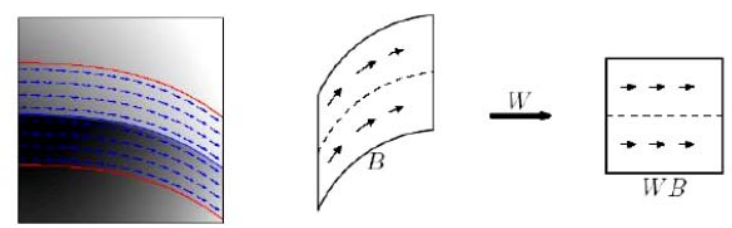

Figure 1. Deformation of horizontal field according to a geometric flow.

\section{A. Flow-curve relationship}

There is a constant correspondence between the flow along the vertical direction and curves whose tangent is never vertical; the flow associated with this curve is given by flow:

$$
\tau(x)=\frac{1}{\sqrt{1+\left|c^{\prime}(x)\right|^{2}}}\left(\begin{array}{l}
1 \\
c^{\prime}(x)
\end{array}\right)
$$

\section{$c^{\prime}(x)$ : Slope of optical flow.}

We can generate the basic test bandelet according to the flow and geometric regularity of each sub-block. If there is no flow geometry in the sub-blocks, this means that the sub-block is uniformly regular so that we can use the classical separable wavelet basis for treating this subblock. If not, the sub-block must be processed by the bandelet.

If the sub-block is not uniformly regular, we can deform it, has variation along geometric flow defined in the sub-block. [12].

For have the sub-block that contains the singularity that is with a calculation of Lagrangian [13].

$$
L(f, R, B)=\left\|f-f_{R}\right\|^{2}+\lambda . T^{2} \sum_{j}\left(R_{j G}+R_{j B}\right)
$$

\section{$\lambda$ : Lagrangian.}

$T$ : Quantifiction step.

$R_{j G}$ : is the number of bits to code the optical flew in each square.

$R_{j B}$ : is the number of bits to code the quantized bandelet coefficients.

\section{B. Quadtree segmentatin}

Segmentation operation it is a division successive of image space that allows us to have a set of subblocks.

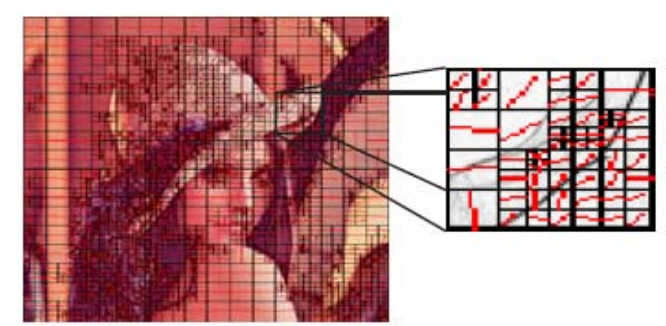

Figure 2. Example of quadtree segmentation
The operating diagram of the quadtree segmentation is the following

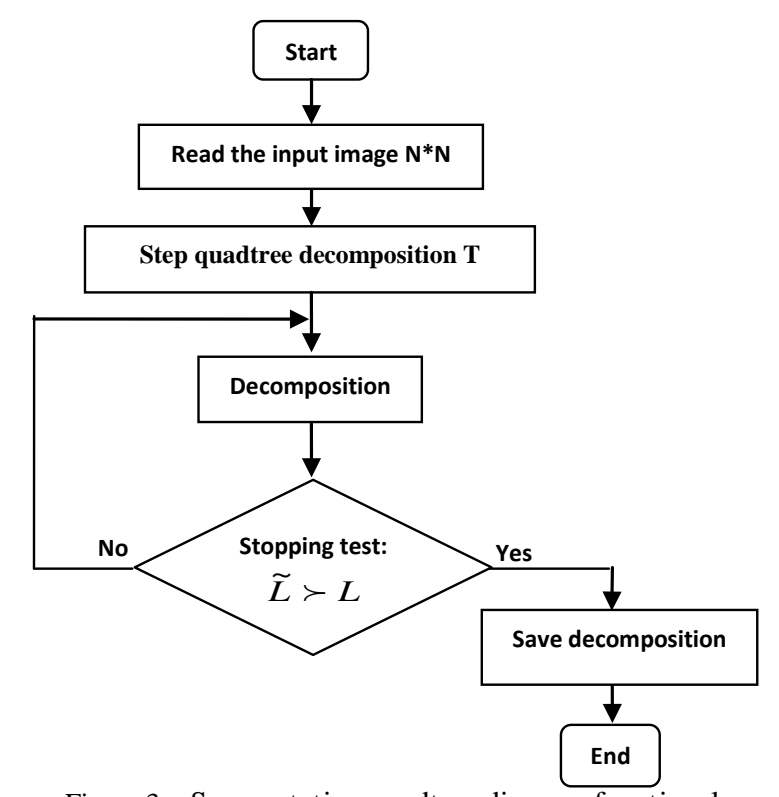

Figure 3. Segmentation quadtree diagram functional

For have the optimal segmentation that is with [14]:

$$
L_{0}(S)=\min \left\{L_{0}(S), \tilde{L}(S)\right\}
$$

Where

$$
\begin{aligned}
& \tilde{L}(S)=L_{0}\left(S_{1}\right)+L_{0}\left(S_{2}\right)+L_{0}\left(S_{3}\right)+L_{0}\left(S_{4}\right)+L_{0}(S)+\lambda \cdot T^{2} \\
& L_{0}\left(S_{i}\right): \text { Lag rangian of sub-blocks. }
\end{aligned}
$$

\section{The operator of deformation}

The deformation operation is a local operation on a block that contains a curve singularity to align or correct in a direction horizontal and vertical.

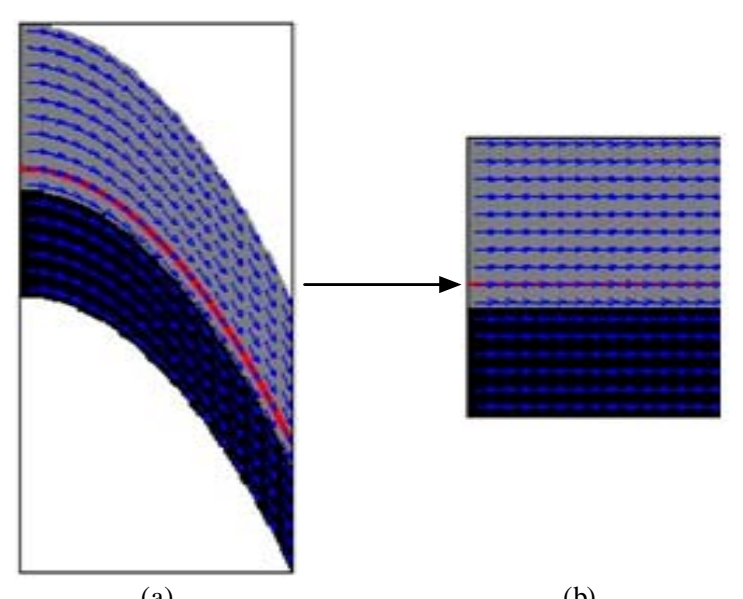

(a)

(b) 


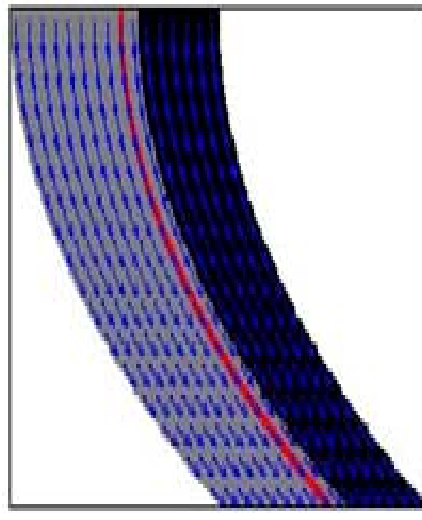

(c)

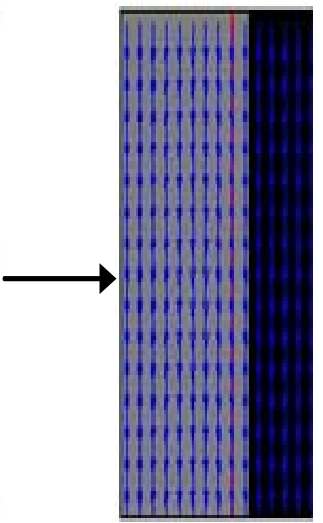

(d)
Figure 4. Example of model horizon and deformation of the field according to a geometric flow. Image distortion: (a) an image having a horizontal flow, (b) its image by the operator $\mathrm{W}$,

(c) an image having a vertical flow, (d) the image by the operator W.

Deformation operation gives a wavelet orthonormal basis of $L^{2}(\Omega)$ :

$$
\left\{\begin{array}{l}
\phi_{j, n_{1}}\left(x_{1}\right) \psi_{j, n_{2}}\left(x_{2}-c\left(x_{1}\right)\right) \\
\psi_{j, n_{1}}\left(x_{1}\right) \phi_{j, n_{2}}\left(x_{2}-c\left(x_{1}\right)\right) \\
\psi_{j, n_{1}}\left(x_{1}\right) \psi_{j, n_{2}}\left(x_{2}-c\left(x_{1}\right)\right)
\end{array}\right\}
$$

The horizontal wavelet $\psi_{j, n}^{H}$ have not vanishing mo ments along contour, to be replaced by new functions:

$\psi_{l, n_{1}}\left(x_{1}\right) \psi_{j, n_{2}}\left(x_{2}-c\left(x_{1}\right)\right)$

This is called bandeletization [15], [16]. The orthonormal basis of bandelet of field warping is defined by:

$$
\left.\left(\begin{array}{l}
\psi_{l, n_{1}}\left(x_{1}\right) \psi_{j, n_{2}}\left(x_{2}-c\left(x_{1}\right)\right) \\
\psi_{j, n_{1}}\left(x_{1}\right) \phi_{j, n_{2}}\left(x_{2}-c\left(x_{1}\right)\right) \\
\psi_{j, n_{1}}\left(x_{1}\right) \psi_{j, n_{2}}\left(x_{2}-c\left(x_{1}\right)\right)
\end{array}\right)=\left\{\begin{array}{l}
\psi_{l, n}^{H} \\
\psi_{j, n}^{V} \\
\psi_{j, n}^{D}
\end{array}\right\} j, l\right\rangle n_{1}, n_{2}
$$

\section{OPERATIONAL DIA GRAM}

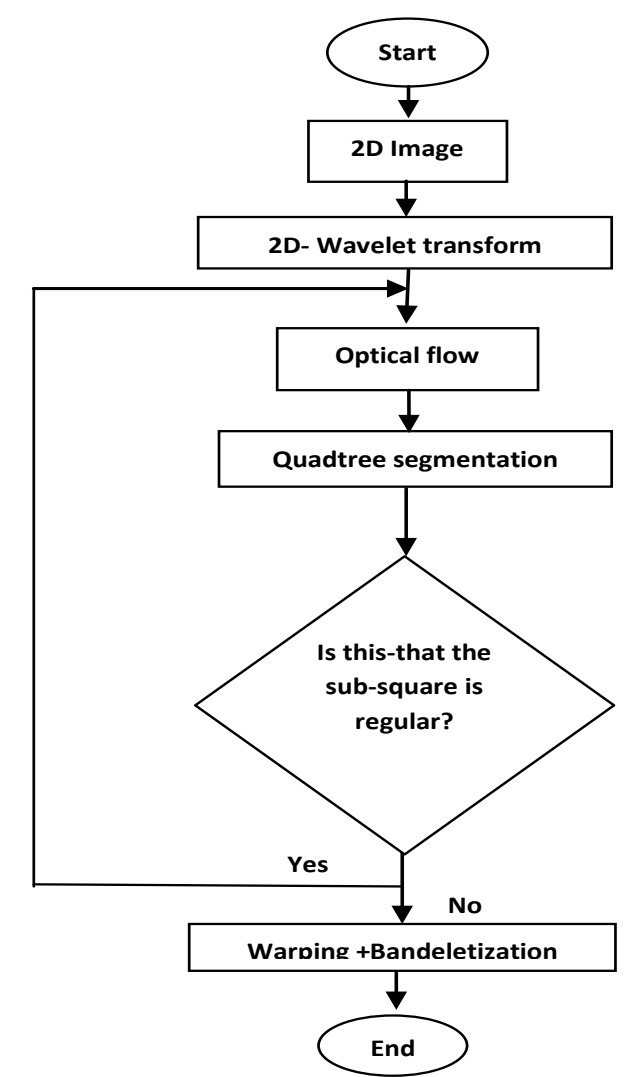

Figure 5. Bandelet transform operational diagram

\section{EZW CODING SCHEME}

The algorithm proposed by Shapiro [17], the design of this algorithm is based on two pass with a calculated threshold $\mathrm{T}$, we determine the maximum value of the wavelet coefficients, so in the first dominant pass, if the coefficient absolute value is greater than the threshold, so we obtained a positive code or negative code according to the sign of coefficient, if the absolute value is less, we check the descendants coefficient, if you have a coefficient with absolute value above the threshold, we obtained IZ code, else we have a code ZT.

In the second pass, the comparison is made in the middle of the interval [Tn, 2Tn[, if this coefficient belongs to the first interval, so we are a transmission of the bit 1 , if this coefficient belongs to the second interval, so there are a transmission of bit 0 .

\section{SPIHT CODING SCHEME}

The SPIHT algorithm proposed by Said and Pearlman in 1996 [18], ameliorate progressive algorithm is compared to the EZW algorithm, based on the creation of three list SCL, ICL and ISL with a calculated threshold T, each time you make a scan on both lists SCL and ISL and that for the classified the significant coefficient in the list of significant coefficient. 


\section{QUALITY EVALUATION PARAMETER}

The Peak Signal to Noise Ratio (PSNR) is the most commonly used as a measure of quality of reconstruction in image compression. The PSNR were identified using the following formulate:

$$
M S E=\frac{1}{M . N} \cdot \sum_{i=0}^{M-1 N-1} \sum_{j=0}[I(i, j)-\hat{I}(i, j)]^{2}
$$

Mean Square Error (MSE) which requires two $\mathrm{MxN}$ gray scale images $I$ and $\hat{I}$ where one of the images is considered as a compression of the other is defined as:

- The PSNR is defined as:

$$
P S N R=10 \cdot \log _{10} \frac{\left(2^{R}-1\right)^{2}}{M S E}[d B]
$$

- The structural similarity index (SSIM):

The PSNR measurement gives a numerical value on the damage, but it does not describe its type. Moreover, as is often noted in [20], [21], it does not quite represent the quality perceived by human observers. For medical imaging applications where images are degraded must eventually be examined by experts, traditional evaluation remains insufficient. For this reason, objective approaches are needed to assess the medical imaging quality. We then evaluate a new paradig $m$ to estimate the quality of medical images, specifically the ones compressed by wavelet transform, based on the assumption that the human visual system(HVS) is highly adapted to extract structural information. The similarity compares the brightness, contrast and structure between each pair of vectors, where the structural similarity index (SSIM) between two signals $\mathrm{x}$ and $\mathrm{y}$ is given by the following expression:

$$
\operatorname{SSIM}(x, y)=l(x, y) c(x, y) s(x, y)
$$

Finally the quality measurement can provide a spatial map of the local image quality, which provides more information on the image quality degradation, which is useful in medical imaging applications. For application, we require a single overall measurement of the whole image quality that is given by the following formula:

$$
\operatorname{MSSIM}(I, \hat{I})=\frac{1}{M} \sum_{i=1}^{M} \operatorname{SSIM}\left(I_{i}, \hat{I}_{i}\right)
$$

Where $I$ and $\hat{I}$ are respectively the reference and degraded images, $I_{i}$ and $\hat{I}_{i}$ are the contents of images at the i-th local window.

$\mathrm{M}$ : the total number of local windows in image. The MSSIM values exhibit greater consistency with the visual quality.

\section{RESULTS AND DISCUSSION}

We are interested in this work to the medical images compression, that we applied algorithm (DBT+SPIHT), $(\mathrm{DBT}+\mathrm{EZW}),(\mathrm{DWT}+\mathrm{SPIHT})$ and $(\mathrm{DWT}+\mathrm{EZW})$. For this, we chose sets of medical images (MRI, CT and ECHO) images gray level size $512 \times 512$ encoded on 8 bits per pixel. These images are taken from the GE Medical System (database) [21]. The importance of our work lies in the possibility of reducing the rates for which the image quality remains acceptable. Estimates and judgments of the compressed image quality are given by the PSNR evaluation parameters and the MSSIM similarity Index.

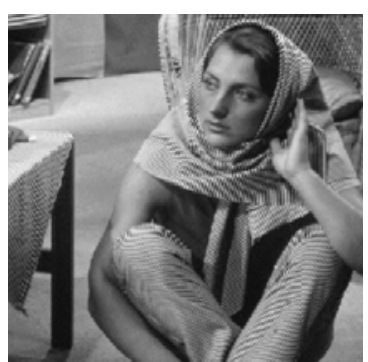

(a)

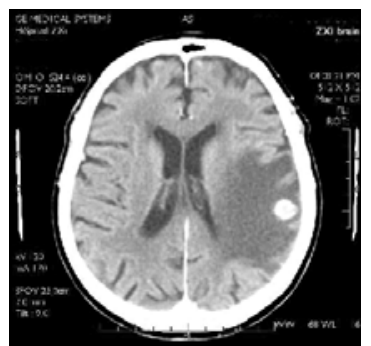

(c)

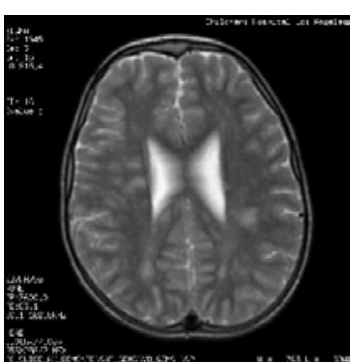

(b)

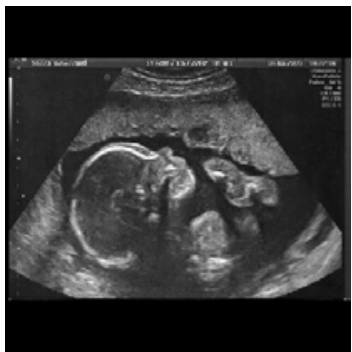

(d)
Figure 6. (a). BARBARA, (b). MRI, (c). CT and (d). ECHO original image

From our investigating, we would highlight that the quality of compressed image depends also on the number of decompositions level, for th is we have try to determine decomposition levels who permits us to have a high value PSNR for two algorithms (DWT+EZW) and (DWT+SPIHT). 

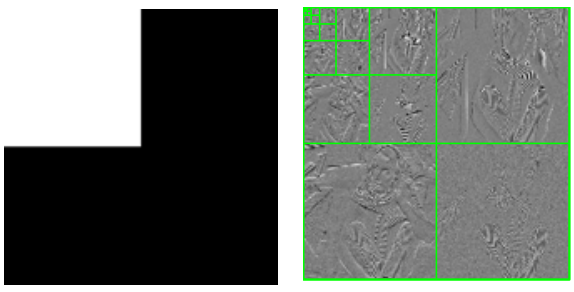

(a). Level-1
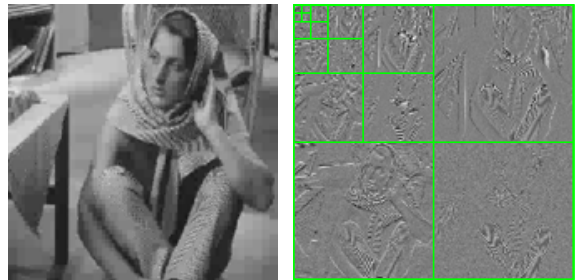

(d) Level-4
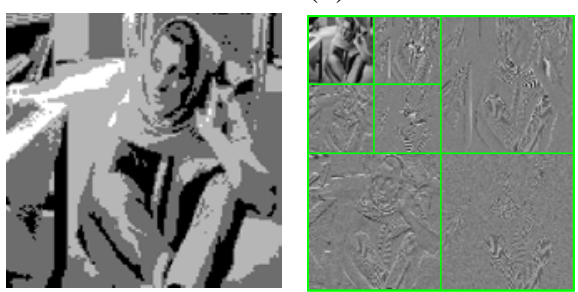

(g) Level-7
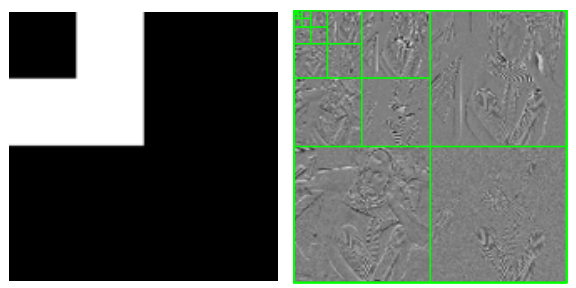

(b). Level-2
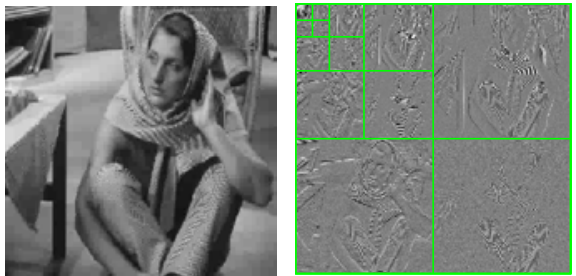

(e) Level-5
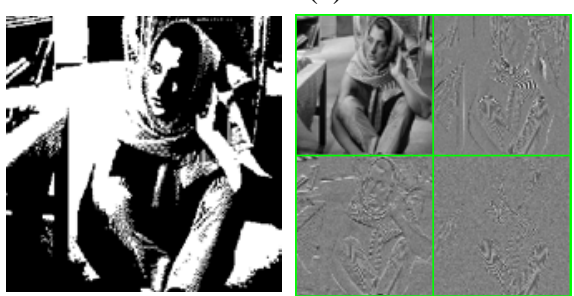

(h) Level-8
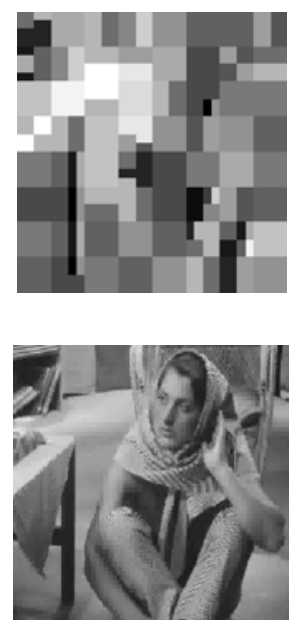

(c). Level-3

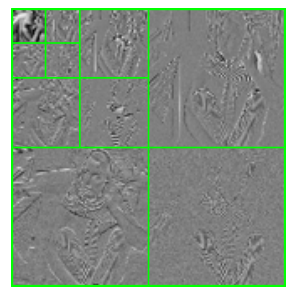

(f) Level-6
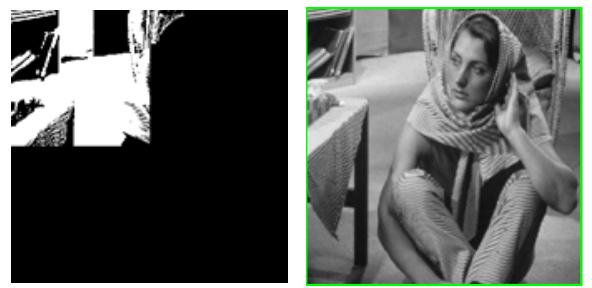

(i) Level-9

Figure 7. Image compressed resultats by (DWT+EZW) for different valus decomposition levels

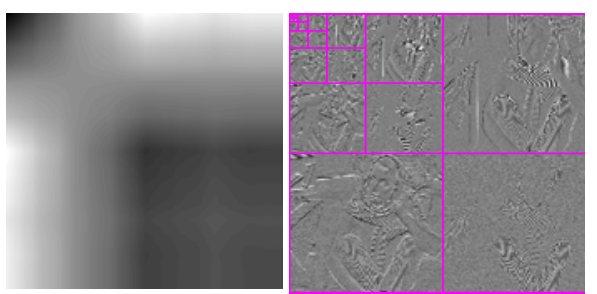

(a). Level-1

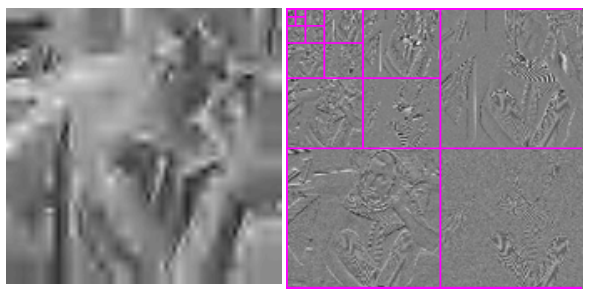

(d) Level-4

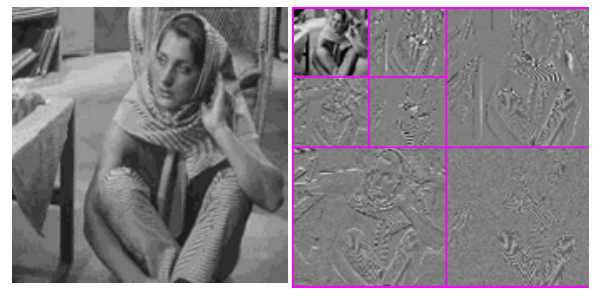

(g) Level -7
Figure 8. Image compressed resultats by (DWT+SPIHT) for

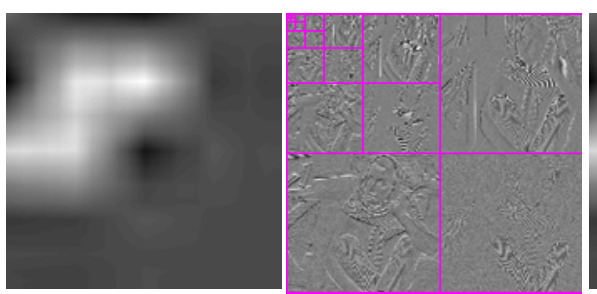

(b). Level-2

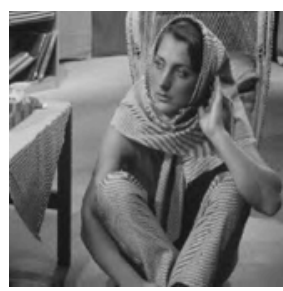

(e) Level -5

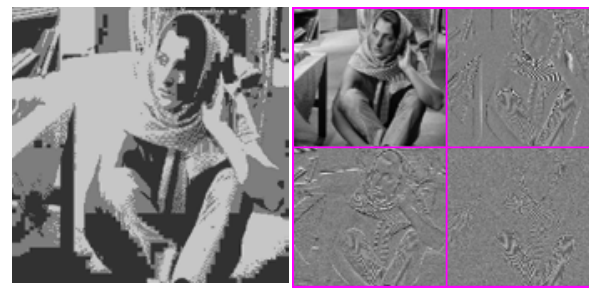

(h) Level-8

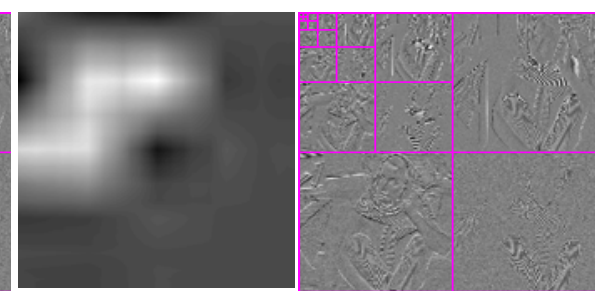

(c). Level -3

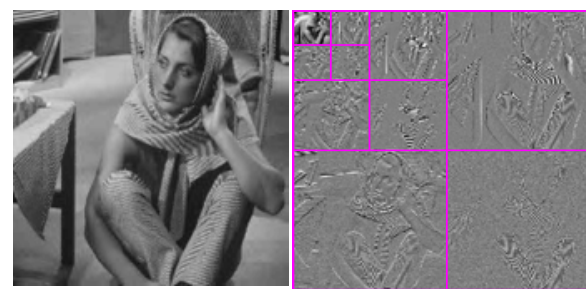

(f) Level-6

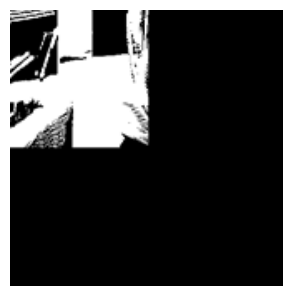

(i) Level-9

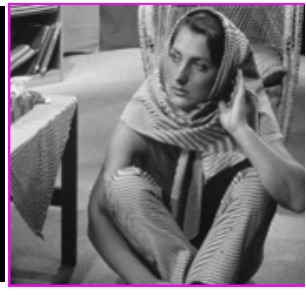




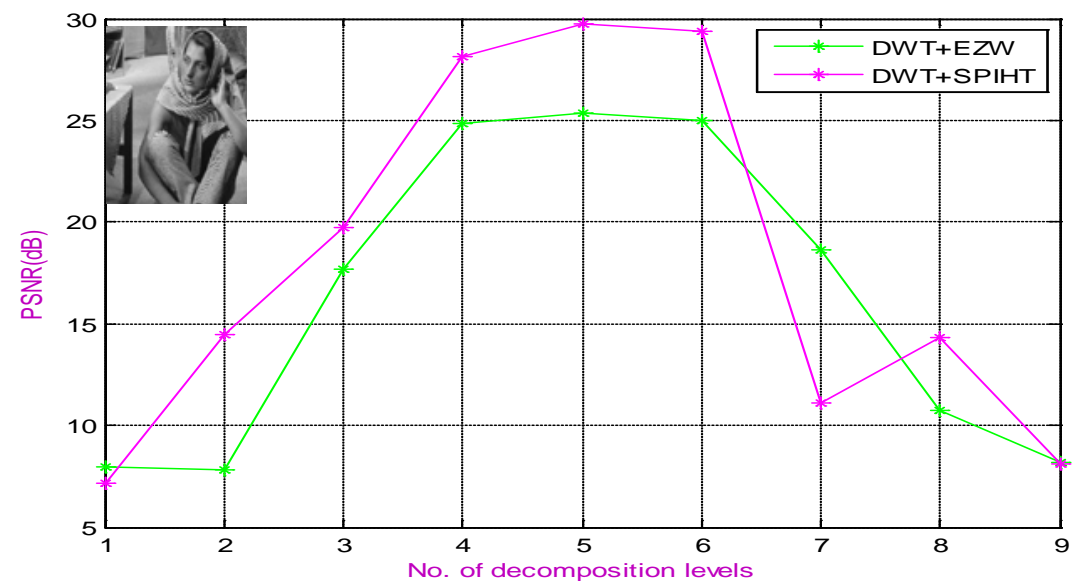

Figure 9. No. of decomposition levels verses PSNR (dB)

It can be seen from figure 9, that we have a high PSNR value for level decomposition equal to five, for this value we presented in the following the results comparison for compressed images by (DBT + SPIHT), $(\mathrm{DBT}+\mathrm{EZW}),(\mathrm{DWT}+\mathrm{SPIHT})$ and $(\mathrm{DWT}+\mathrm{EZW})$ algorith ms.
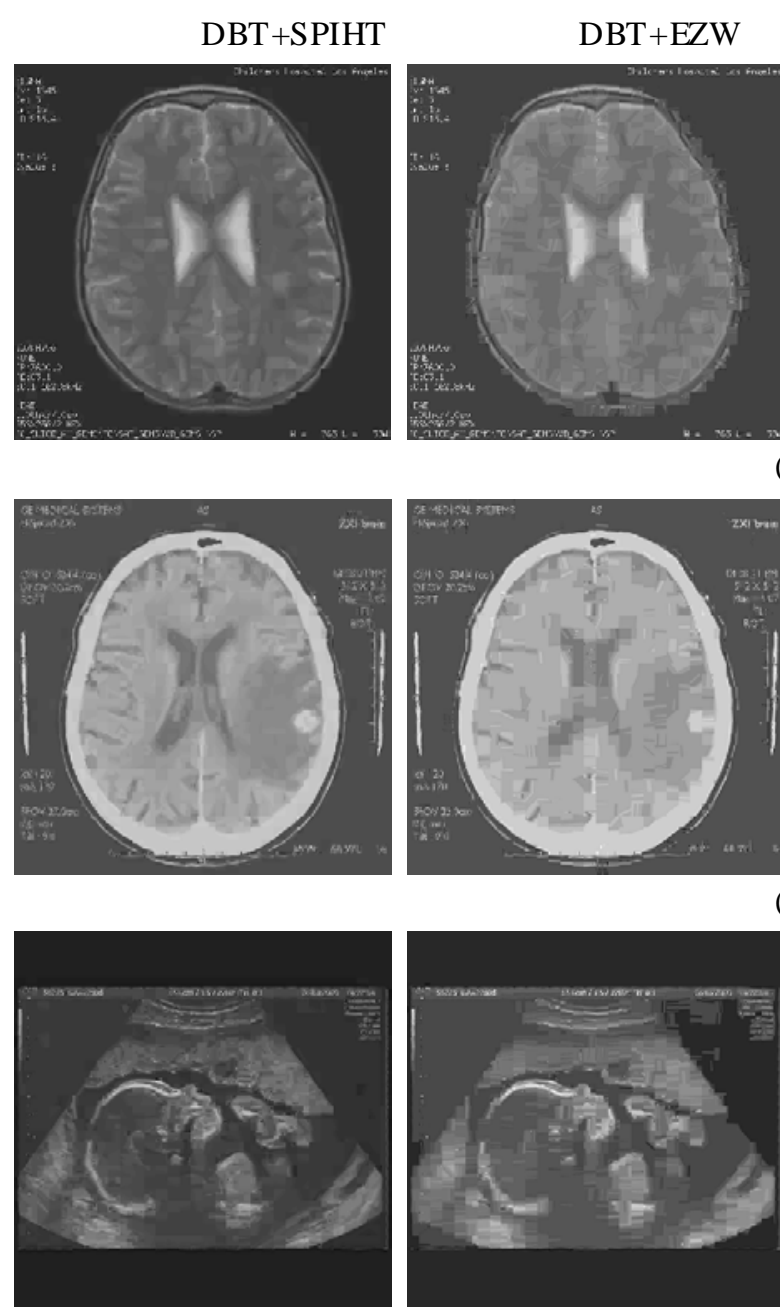

(a)
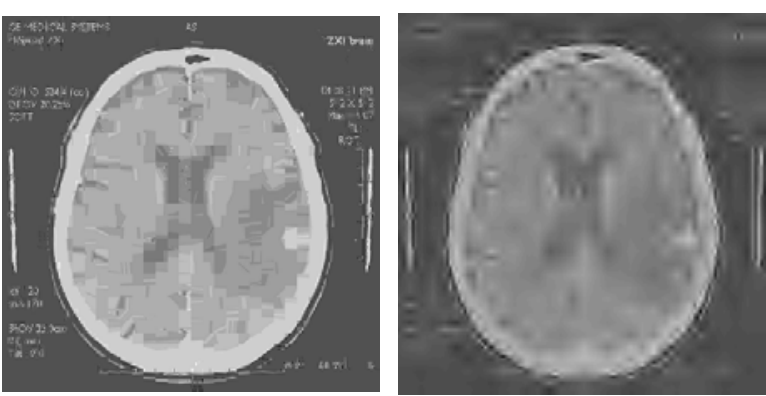

(b)
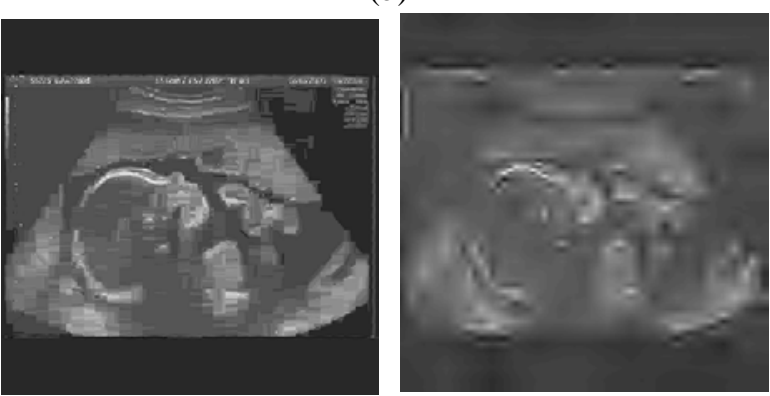

(c)

DWT+EZW
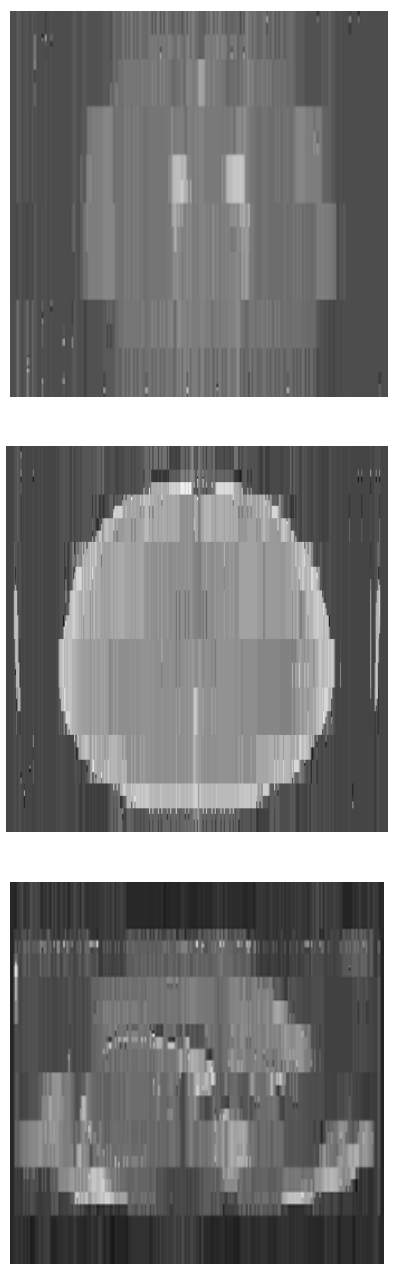

Figure 10. MRI, CT and ECHO compressed images by (DBT + SPIHT), (DBT + EZW), (DWT + SPIHT) and (DWT + EZW) algorithm for $R_{c}=0.5 b p p$ : (a) MRI image, (b) CT image, (c) ECHO image 

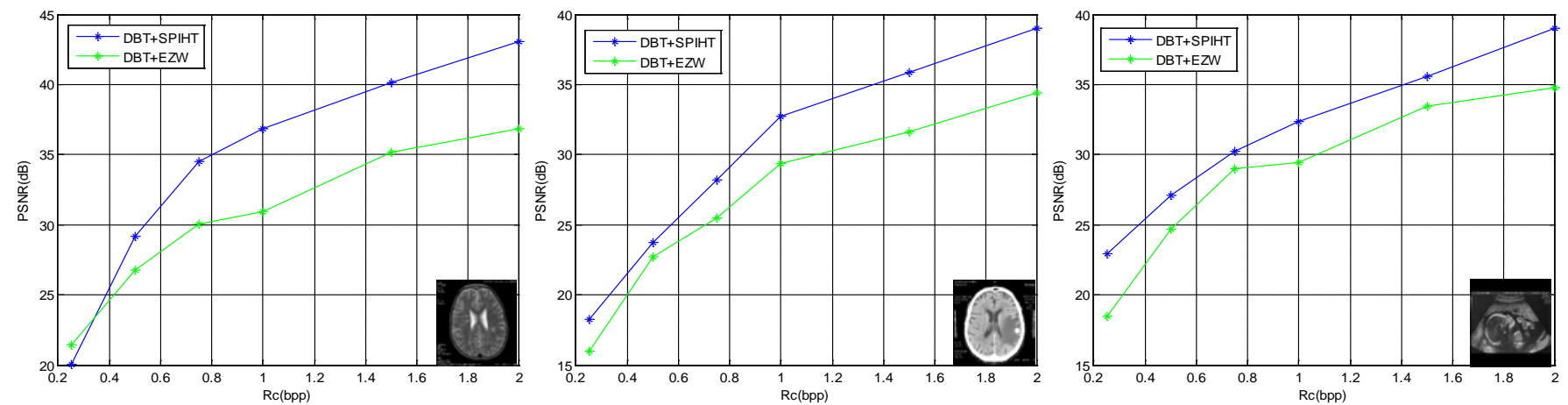

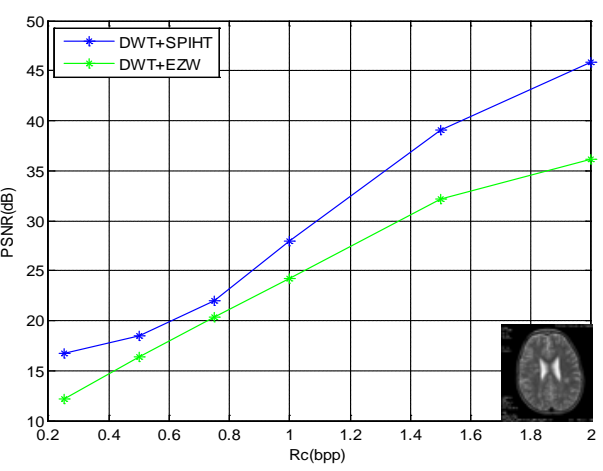

(a)

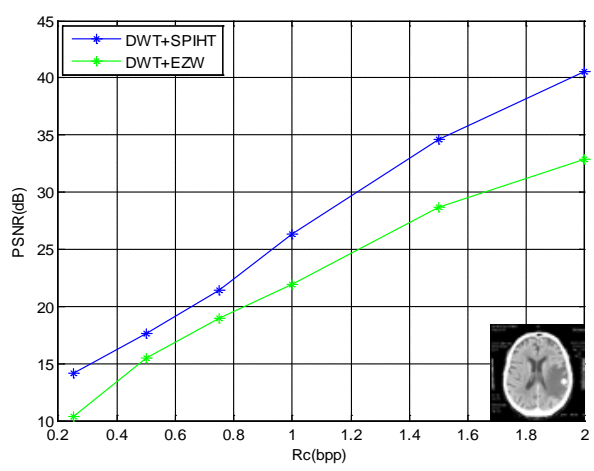

(b)

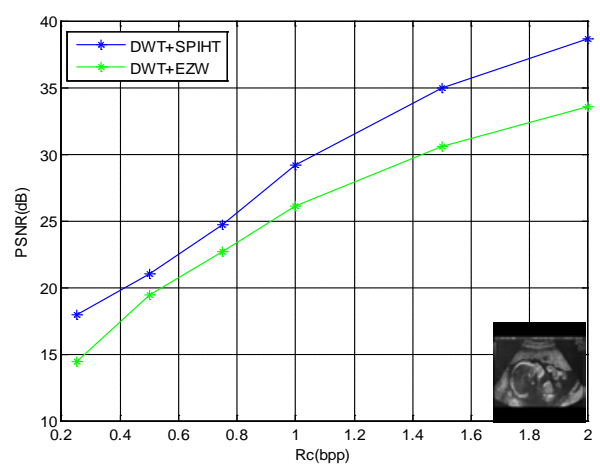

(c)

Figure 11. Numerical results PSNR of compression images by bandelet and wavelet transform coupled with SPIHT and EZW coder: (a) MRI image, (b) CT image, (c) ECHO image
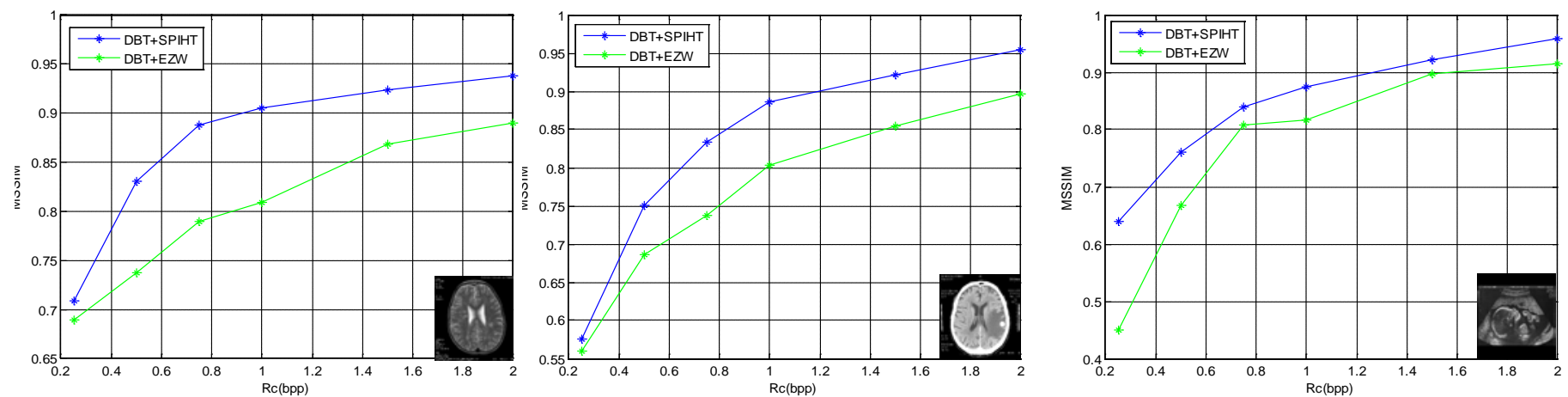

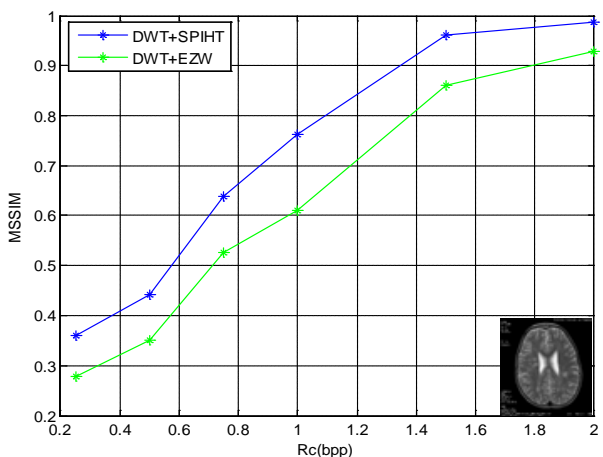

(d)

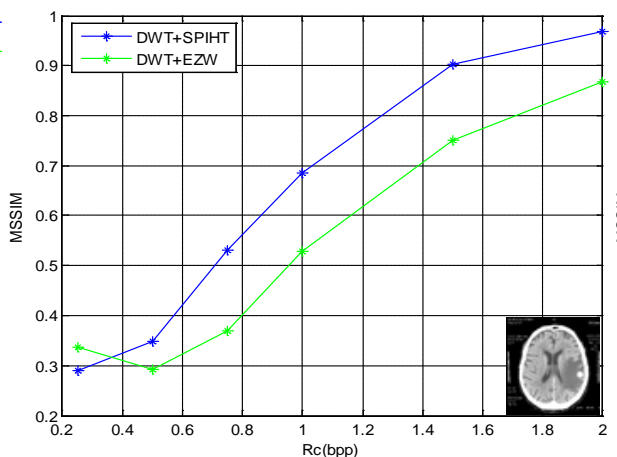

(e)

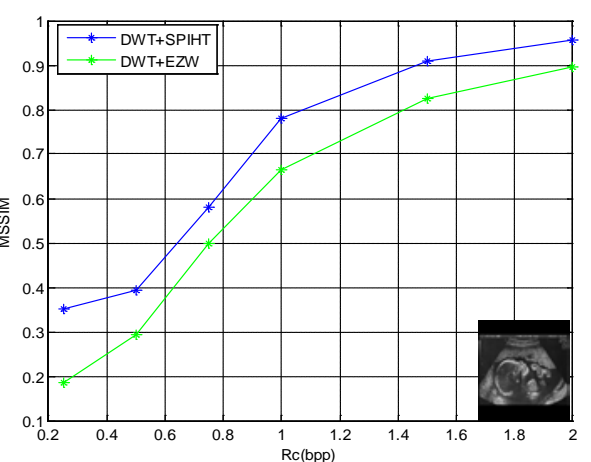

(f)

Figure 12. Numerical results MSSIM of compression images by bandelet and wavelet transform coupled with SPIHT and EZW coder :

(d) MRI image, (e) CT image, (f) ECHO image

We see from the figure 11 and 12, that the bandelet transform coupled with SPIHT coder offer PSNR and MSSIM values better than to the other algorithms $(\mathrm{DBT}+\mathrm{EZW})(\mathrm{DWT}+\mathrm{SPIHT}),(\mathrm{DWT}+\mathrm{EZW})$.
In order to specify the type of medical image adapted to algorithm (DBT+SPIHT), we recapitulate the results for the three medical images (MRI, CT and ECHO) 
compressed by (DBT+SPIHT) algorith $\mathrm{m}$ in the following figure.

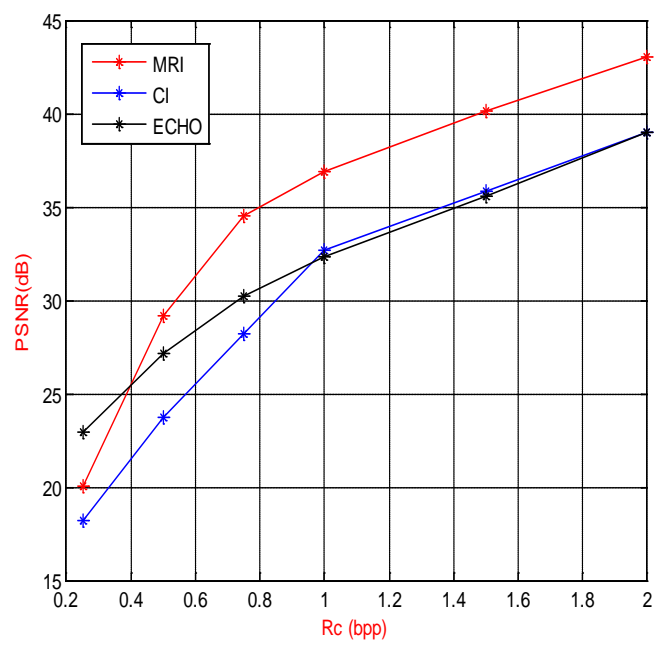

Figure 13. Comparison results between MRI, CT and ECHO image compressed by bandelet transform coupled by SPIHT coder

By comparing the different PSNR values, we can say that our algorithm (DBT+SPIHT) is adapted to the MRI images compression.

After displaying the performance of the compression bandelet to medical images, now we apply our compression algorith $m$ to the satellite image compression. To do so, we opted for a satellite image of size $512 \times 512$ (grayscale) encoded on 8bpp.

To investigate the influence of the choice of algorithm, we vary the rate of 0.25 to $2 \mathrm{bpp}$, and calculate the evaluation parameters (PSNR, MSSIM) for both algorithms based on bandelet transform. The results are given in the following figure.

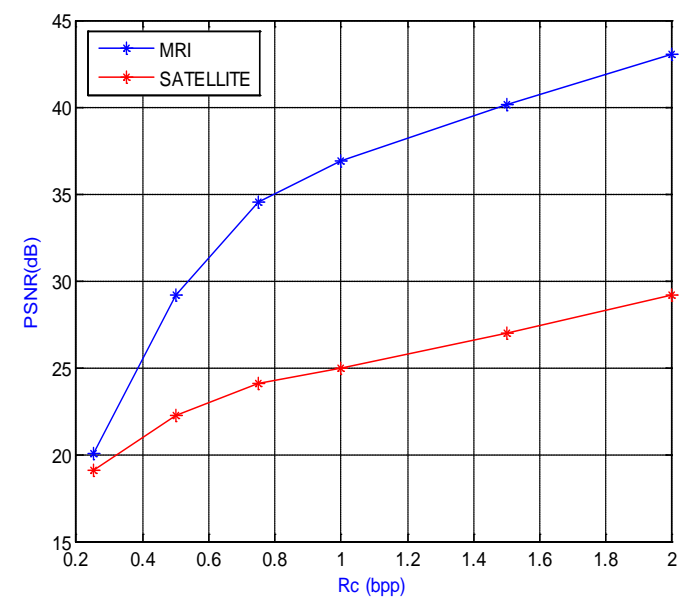

Figure 14. Compression results for MRI and Satellite image compressed by (DBT+SPIHT) algorithm

Visually, from the two curves, it is clearly that the (DBT +SPIHT) algorithm allows us to have a good image reconstruction so a better image visual quality and this is proved by the large values of the parameters evaluation.

\section{CONCLUSION}

Medical image compression using the bandelet transform is still a vast research field. These transform provides a very compact representation of images and can capture more chaotic geometries and a major improvement compared to other transforms. However, research in this domain is still in its beginning and the current obtained results are difficult to evaluate.

In the future we are going to work into defining a strategy to exploit the bandelet transform for color image compression.

\section{REFERENCES}

[1] M. Nelson. The data compression book. 2nded, M\&T books, New York, 1996.

[2] J. L. Starck, F. Murtagh, E. Candès, and D.L. Donoho. Gray and Color Image Contrast Enhancement by the Curvelet Transform. IEEE Transaction on Image Processing, Vol. 12, No. 6, pp 706-717, 2003.

[3] U E. J. Candès and D. L. Donoho. New Tight Frames of Curvelets and Optimal Representations of Objects with Piecewise C2 Singularities, Comm. on Pure and Appl. Math. Vol. 57, pp.219-266, 2004.

[4] M. N. Do and M. Vetterli. Contourlets, in Beyond Wavelets. J. Stoeckler and G. V. Welland, Eds. San Diego, CA: Academic Press, 2003.

[5] K. Romberg, M.B. Wakin, and R.G. Baraniuk. Approximation and Compression of Piecewise Smooth Images Using a Wavelet/Wedgelet Geometric Model. In IEEE 2003 International Conference on Image Processing -- ICIP-2003, Barcelona, Spain, September 2003.

[6] Borup. Lasse and Nielsen. Morten. Approximation with brushlet systems, Journal of Approximation Theory. Vol. 123, pp. 25-51, 2003.

[7] Vladan Velisavljevi'c, Baltasar Beferull-Lozano, Martin Vetterli and Pier Luigi Dragotti. Directionlets: Anisotropic Multidirectional Representation with Separable Filtering. IEEE Trans. Image Processing, Dec, 2004.

[8] E. Le. Pennec and S. Mallat. Bandelet representation for image compression. In Proc. Int. Conf. Image Processing, Oct. 2001.

[9] E. Le Pennec et S. Mallat. Représentation d'images par bandelettes et application à la compression. Dans GRETSI, Toulouse, Septemb re 2001.

[10] E. Le Pennec. Bandelettes et représentation géométrique des images. Thèse de Doctorat, Ecole Polytechnique, 19 décemb re 2002.

[11] V. Chappelier. Codage progressif d'images par ondelettes orientées. Thèse de Doctorat, Université Rennes 1, 15 décemb re 2005.

[12] Ding. Time Frequency Analysis Tutorial. R99942057.

[13] Hong Han,Xing Wu. Research of Method in Human Detection Based on Bandelet Transform. Laboratory 
of Intelligent Perception and Image Understanding of Ministry of Education of China, Vol. 74951S,2009.

[14] Ruihua Liang, Lizhi Cheng, Zhicheng Zhu, Bo Chen. Image coding based on second generation bandelet transform. Modern electronics technique, 61-65 (2007).

[15] E. Le Pennec, S. Mallat. Bandelettes et représentation géométrique des images. CMAP / école Polytechnique 91128 PALAISEAU - France.

[16] E. Le Pennec, S. Mallat. Bandelet Representations For Image Compression. 0-7803-6725-1/01. IEEE. 2001.

[17] J.SHAPIRO. Embedded Image Coding using Zerotree of Wavelet Coefficients. IEEE trans. Signal processing. Vol. 41, pp. 3445-3462. Dec, 1993.

[18] A. Said and W. A. Pearlman (1996). A new and efficient image codec based on set partitioning in hierarchical trees. IEEE Transactions on Circuits and Systems for Video Tech., vol. 6, pp. 243-250.

[19] W.S. Geisler, M.S. Banks. Visual Performance. Handbook of Optics - Vol. 1, McGraw-Hill, NY, USA, 1995.

[20] A.B. Watson, L.B. Kreslake. Measurement of Visual Impairment Scales for Digital Video. Human Vision and Electronic Imaging Conference, San Jose, CA, USA, SPIE Vol. 4299, Jan. 2001, 2001, pp. 79 - 89.

[21] www.GE Medical System.com (database).

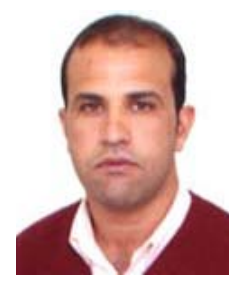

Mohammed BELADGHAM was born in Tlemcen, Algeria; he's received the electrical engineering diploma from university of Tlemcen, Algeria, and then a Magister in signals and systems from university of Tlemcen, Algeria and the Ph.D. degree in Electronics from the University of Tlemcen (Algeria), in 2012. His research interests are Image processing, Medical image compression, wavelets transform and optimal encoder.

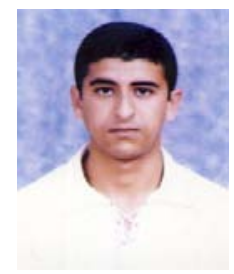

Yassine HABCHI was born in Mechria, A lgeria, received his Bachelor (2005) and Engineers (2010) degree in Electrical Engineering from Saïda University in Algeria, and his Magister (2013) degree in Electronic from university of Bechar, Algeria. His current research interest includes Image processing, Medical image compression, wavelets transform and optimal encoder.

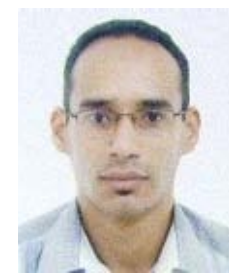

A. MOULAY LAKHDAR I got my Engineering Degree in Telecommunication in 2000 at the Institute of Telecommunications in Oran. Magister was my second degree in Signal and telecom at Djillali LIABES university of Sidi Bel Abbes in 2003. From 2004 to this day I work in the Bechar University as lecturer. Since May 2009, I graduated PhD Es Sciences at the of Sidi Bel Abbes. I do my research at the Bechar University and Communications, Architecture and Media Laboratory (CAMR) (Djillali LIABES University). His research interests are Image transmission, Image processing, and digital transmission performances.

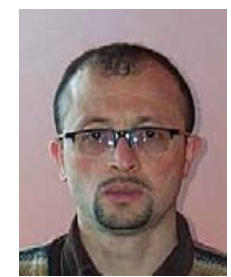

Abdelmalik TALEB-AHMED was born in Roubaix, France, in 1962. He received a post graduate degree and a $\mathrm{Ph}$. D. in Electronics and Microwaves from the University of Lille 1 in 1988 and 1992. From 1992 to 2004, He was an Associate Professor at the University of Littoral, Calais. Since 2004, He is currently a Professor at the University of Valenciennes in the department GE2I, and does his research at the LAMIH FRE CNRS 3304 UVHC, His research interests includes signal and image processing. Image segmentation, Prior knowledge integration in image analysis, Partial Differential Equations and Variational Methods in image analysis, Image compression, Multimodal signal processing, Medical image analysis, including mu ltimodal image reg is tration, etc. 